\title{
Uncertainty and sensitivity analysis of fission gas behavior in engineering-scale fuel modeling
}

\author{
G. Pastore ${ }^{\mathrm{a}, *}$, L.P. Swiler ${ }^{\mathrm{b}}$, J.D. Hales ${ }^{\mathrm{a}}$, S.R. Novascone $^{\mathrm{a}}$, D.M. Perez ${ }^{\mathrm{a}}$, B.W. Spencer ${ }^{\mathrm{a}}$, L. Luzzi ${ }^{\mathrm{c}}$, P. Van Uffelen ${ }^{\mathrm{d}}$, \\ R.L. Williamson ${ }^{\mathrm{a}}$ \\ ${ }^{a}$ Fuel Modeling and Simulation, Idaho National Laboratory, P.O. Box 1625, Idaho Falls, ID 83415-3840, United States \\ ${ }^{b}$ Optimization and Uncertainty Quantification, Sandia National Laboratories, P.O. Box 5800, Albuquerque, NM 87185-1318, United States \\ ${ }^{c}$ Politecnico di Milano, Department of Energy, Nuclear Engineering Division, via La Masa 34, I-20156 Milano, Italy \\ ${ }^{d}$ European Commission, Joint Research Centre, Institute for Transuranium Elements, Hermann-von-Helmholtz-Platz 1, D-76344 \\ Karlsruhe, Germany
}

\begin{abstract}
The role of uncertainties in fission gas behavior calculations as part of engineering-scale nuclear fuel modeling is investigated using the BISON fuel performance code with a recently implemented physics-based model for fission gas release and swelling. Through the integration of BISON with the DAKOTA software, a sensitivity analysis of the results to selected model parameters is carried out based on $\mathrm{UO}_{2}$ single-pellet simulations covering different power regimes. The parameters are varied within ranges representative of the relative uncertainties and consistent with the information in the open literature. The study leads to an initial quantitative assessment of the uncertainty in fission gas behavior predictions with the parameter characterization presently available. Also, the relative importance of the single parameters is evaluated. Moreover, a sensitivity analysis is carried out based on simulations of a fuel rod irradiation experiment, pointing out a significant impact of the considered uncertainties on the calculated fission gas release and cladding diametral strain. The results of the study indicate that the commonly accepted deviation between calculated and measured fission gas release by a factor of 2 approximately corresponds to the inherent modeling uncertainty at high fission gas release. Nevertheless, significantly higher deviations may be expected for values around $10 \%$ and lower. Implications are discussed in terms of directions of research for the improved modeling of fission gas behavior for engineering purposes.
\end{abstract}

Keywords: nuclear fuel, fuel modeling, fission gas behavior, uncertainty, sensitivity analysis

\section{Introduction}

The complex behavior of the fission gases xenon and krypton in $\mathrm{UO}_{2}$ significantly affects the thermo-mechanical performance of the nuclear fuel rods employed in current light water reactors (LWR). Gas retention in the form of bubbles leads to fuel swelling which promotes pelletcladding mechanical interaction (PCMI), and the concomitant fission gas release (FGR) to the fuel rod free volume increases the rod internal pressure, both processes affecting the mechanical behavior of the cladding. Moreover, gas release and precipitation in bubbles affect the thermal conductance of the fuel-cladding gap and the fuel thermal conductivity, respectively, and consequently the temperature distribution in the fuel pellet $[1,2]$. Hence, the confidence in fuel thermo-mechanics calculations by means of

\footnotetext{
* Corresponding author

Email addresses: Giovanni.Pastore@inl.gov (G. Pastore), LPSwile@sandia.gov (L.P. Swiler), Jason.Hales@inl.gov (J.D. Hales), Stephen. Novascone@inl.gov (S.R. Novascone), Danielle.Perez@inl.gov (D.M. Perez), Benjamin.Spencer@inl.gov (B.W. Spencer), Lelio.Luzzi@polimi.it (L. Luzzi), Paul.Van-Uffelen@ec.europa.eu (P. Van Uffelen), Richard.Williamson@inl.gov (R.L. Williamson)
}

fuel performance codes partly depends on the confidence in fission gas behavior modeling.

The computational analysis of fission gas release and swelling involves the numerical treatment of multiple intricate and mutually dependent phenomena, and inevitably depends on uncertain parameters. Indeed, many internal parameters of fission gas behavior models are difficult to measure and prone to large uncertainties. In addition, the dependency of the relevant processes on the general solution variables (e.g., temperature) implies that uncertainties pertaining to the global fuel analysis turn into uncertainties in fission gas behavior calculations. International benchmarks of fuel performance codes [3-5] proved that difficulties still remain with predicting FGR and fuel dimensional changes due to swelling, and that given identical conditions, different models provide a wide spectrum of predictions. This can be ascribed to the complexity and non-linearity of the physical processes involved, as well as to the uncertainties pertaining to the model parameters.

Lassmann and Benk [6] noted that numerical errors associated with algorithms presently used to analyze fission gas diffusion are outweighed by parameter uncertainties, including uncertainties in the calculated fuel temperature 
and grain size. Olander and Van Uffelen [7] pointed out the high uncertainty in the atomic diffusion coefficients and the relative impact on mechanistic fission gas behavior calculations, as well as the general lack of recognition of this point. Van Uffelen [8] and Lösönen [9] demonstrated the significant sensitivity of FGR calculations to uncertain parameters such as temperature, intra-granular and grain-boundary diffusion coefficients, and fuel grain radius, in a systematic manner. As of today, a deviation between calculated and measured FGR within a factor of 2 is commonly accepted for fuel performance codes $[3-5,10]$. Nevertheless, this is effectively a convention rather than the accuracy considered as attainable on the grounds of systematic uncertainty evaluation studies.

This paper presents a systematic sensitivity analysis of fission gas behavior to selected uncertain parameters. Modeling of fission gas release and swelling as part of a global engineering-scale fuel thermo-mechanical analysis $[2,11]$ is considered. The aim of the work is to provide an initial quantitative assessment of the uncertainty in fission gas behavior predictions with the parameter characterization presently available, as well as to identify the most important sources of results variability. The number of considered parameters is limited and the relative sensitivity ranges are approximate, in a simple approach intended as a first step of uncertainty evaluation.

For the purpose of obtaining correct sensitivity of the results to various physical parameters and in a broad range of conditions, physics-based models of the complex phenomena occurring in nuclear fuel during irradiation bring substantial advantages compared to empirical correlations $[12,13]$. An efficient physics-based model of fission gas release and swelling in $\mathrm{LWR}-\mathrm{UO}_{2}$ fuel was recently developed for the TRANSURANUS fuel performance code [14] at Politecnico di Milano and at Institute for Transuranium Elements of the European Commission [15]. Based on this TRANSURANUS development, a new model was subsequently implemented in the BISON finite-element fuel performance code developed at Idaho National Laboratory (INL) [16]. In this work, the BISON code with the new physics-based model of fission gas behavior, and the DAKOTA uncertainty quantification and sensitivity analysis software developed at Sandia National Laboratories [17] are used.

The outline of the paper is as follows. Section 2 summarizes the fission gas behavior model used for the study. Section 3 presents the selection of the sensitivity parameters and discusses the relative uncertainties. Section 4 describes the work methodology, including the software framework, the analyzed fuel irradiation cases, and the adopted sensitivity analysis techniques. Section 5 presents and discusses the results of the study. Conclusions are drawn in Section 6.

\section{Fission gas behavior model}

The fundamental physical processes that underlie fission gas behavior in irradiated $\mathrm{UO}_{2}$ may be outlined as follows $[1,2,18-20]$. Fission gas atoms generated in the fuel grains diffuse towards the grain boundaries through repeated trapping in and irradiation-induced resolution from nanometer-size intra-granular bubbles. Gas accumulates at grain boundaries as a result of intra-granular diffusion and by gas sweeping of moving boundaries as the grain growth process takes place. Micron-size grain-face bubbles grow with inflow of gas atoms from within the grains and absorption of vacancies from the grain boundaries, giving rise to grain-face swelling. Bubble growth brings about bubble coalescence and inter-connection, eventually leading to the formation of a tunnel network through which a fraction of the gas is released to the fuel rod free volume (thermal FGR).

The model implemented in the BISON code incorporates the above processes in order to calculate the coupled fission gas release and swelling concurrently. The underlying approach is physics-based but simple, and has been effectively applied to engineering-scale fuel modeling as demonstrated in [15,21-23]. The model features applied in the present work are briefly described in Sections 2.1 and 2.2 .

\subsection{Intra-granular gas diffusion}

Fission gas transport from within the fuel grains (assumed to be spherical) to the grain boundaries is computed via numerical solution of the relevant diffusion equation in one-dimensional spherical geometry

$$
\frac{\partial C_{i g}}{\partial t}=\frac{b}{b+g} D_{i g} \frac{1}{r^{2}} \frac{\partial}{\partial r}\left(r^{2} \frac{\partial C_{i g}}{\partial r}\right)+\beta
$$

where $C_{i g}\left(\mathrm{~m}^{-3}\right)$ is the intra-granular gas concentration, $t(\mathrm{~s})$ the time, $g\left(\mathrm{~s}^{-1}\right)$ the rate of gas atom trapping into intra-granular bubbles (trapping parameter), $b\left(\mathrm{~s}^{-1}\right)$ the rate of gas atom resolution from bubbles back into the lattice (resolution parameter), $D_{i g}\left(\mathrm{~m}^{2} \mathrm{~s}^{-1}\right)$ the intra-granular gas atom diffusion coefficient, $r(\mathrm{~m})$ the radial coordinate in the spherical grain, and $\beta\left(\mathrm{m}^{-3} \mathrm{~s}^{-1}\right)$ the gas generation rate. The term $b /(b+g) D_{i g}$ represents the effective intragranular diffusion coefficient according to Speight [24].

\subsection{Grain-boundary gas behavior}

The grain-boundary gas behavior analysis involves the calculation of both fission gas swelling and thermal release through a direct description of the grain-face bubble development. The major features of the analysis are the following:

- The absorption rate of gas at the grain-face bubbles is assumed to equal the arrival rate of gas at the grain boundaries $[7,19]$. 
- An initial number density of grain-face bubbles is considered, and further nucleation during the irradiation is neglected (one-off nucleation, e.g., [19]).

- All grain-face bubbles are considered to have, at any instant, equal size and equal lenticular shape of circular projection with semi-dihedral angle of $50^{\circ}$ [18,25].

- Grain-face bubble growth (or shrinkage) by inflow of gas atoms from within the grains and concomitant absorption (or emission) of vacancies from the grain boundaries is considered. The bubble growth/shrinkage rate is calculated as

$\frac{d V_{g f}}{d t}=\omega \frac{d n_{g}}{d t}+\Omega \frac{d n_{v}}{d t}$

where $V_{g f}\left(\mathrm{~m}^{3}\right)$ is the bubble volume, $\omega\left(\mathrm{m}^{3}\right)$ the Van der Waals covolume of a fission gas atom, $n_{g}(-)$ the number of fission gas atoms per bubble, $\Omega\left(\mathrm{m}^{3}\right)$ the atomic (vacancy) volume in the bubble, and $n_{v}(-)$ the number of vacancies per bubble. The gas atom inflow rate at the bubble, $d n_{g} / d t$, is obtained from Eq. 1. The vacancy absorption/emission rate at the bubble, $d n_{v} / d t$, is calculated using the model of Speight and Beere [26]

$\frac{d n_{v}}{d t}=\frac{2 \pi D_{g b} \delta_{g b}}{k T S}\left(p-p_{e q}\right)$

where $D_{g b}\left(\mathrm{~m}^{2} \mathrm{~s}^{-1}\right)$ is the vacancy diffusion coefficient along grain boundaries, $\delta_{g b}(\mathrm{~m})$ the thickness of the diffusion layer in grain boundaries, $k\left(\mathrm{JK}^{-1}\right)$ the Boltzmann constant, $T(\mathrm{~K})$ the temperature, and the parameter $S(-)$ depends on the fraction of grain faces covered by bubbles (fractional coverage) as detailed in [19]. The pressure of the gas in the bubble, $p(\mathrm{~Pa})$, is calculated based on the Van der Waals equation of state as [19]

$p=\frac{k T}{\Omega} \frac{n_{g}}{n_{v}}$

The mechanical equilibrium pressure, $p_{e q}(\mathrm{~Pa})$, is given by the sum of the bubble surface tension force and the hydrostatic stress in the surrounding medium.

- Grain-face bubble coalescence is described using an improved model of White $[15,19]$. The variation rate due to coalescence of the bubble number density, $N_{g f}\left(\mathrm{~m}^{-2}\right)$, is calculated as a function of the variation rate of the bubble projected area on the grain face, $A_{g f}\left(\mathrm{~m}^{2}\right)$. The latter is obtained from Eq. 2. A lower limit $N_{g f, l o w}=10^{10}$ $\mathrm{m}^{-2}$ is set, which is based on experimental observations $[19,27]$.

- Fission gas swelling due only to grain-face gas bubbles is considered in this work (e.g., [28,29]). Under the above assumptions, the fractional volume grain-face fission gas swelling is calculated at each time step as

$\frac{\Delta V}{V}=\frac{1}{2} \frac{3}{r_{g r}} N_{g f} V_{g f}$

where $V\left(\mathrm{~m}^{3}\right)$ is the fuel volume, $r_{g r}(\mathrm{~m})$ the grain radius, and $3 / r_{g r}$ represents the grain surface to volume ratio.
- Thermal FGR is modeled based on a principle of grain face saturation. More precisely, after the fractional coverage, $F_{c}(-)$, attains a saturation value, $F_{c, s a t}$, further bubble growth is compensated by gas release in order to maintain the constant coverage condition

$\frac{d F_{c}}{d t}=\frac{d\left(N_{g f} A_{g f}\right)}{d t}=0 \quad$ if $F_{c}=F_{c, s a t}$

This representation allows for the incubation behavior of thermal FGR [30]. Note that fission gas release and swelling are described as inherently coupled phenomena, as fission gas release from the grain faces counteracts bubble growth and thereby fission gas swelling. A limiting situation of swelling saturation may be attained, which corresponds to the condition of constant bubble coverage (Eq. 6) and constant bubble number density at the lower limit mentioned above. This characteristic of the model is consistent with experimental observations demonstrating the saturation tendency of the fission gas swelling [31].

\section{Characterization of the uncertain parameters}

Based on a literature review, five input parameters of the fission gas behavior model are selected and approximate uncertainty ranges are estimated. The uncertainty is interpreted as the 95\% confidence interval of the parameter distribution. Details are given in Sections 3.1 to 3.5 .

\subsection{Temperature}

Various mechanisms affecting fission gas behavior are temperature-dependent. Therefore, fission gas behavior modeling applied to fuel performance codes is affected by uncertainties in the calculated fuel temperature, in addition to those pertaining to internal model parameters. Moreover, temperature uncertainty combines with the intrinsic uncertainty of temperature-dependent parameters. In fact, the scatter between fuel temperature predictions by different fuel performance codes has been observed to be amplified in the scatter of FGR predictions [3]. According to the analyses of Bernard et al. [10], a $5 \%$ variation of temperature could lead to a $30 \%$ variation of the calculated FGR.

The present fission gas behavior model receives the local temperature calculated by the fuel performance code through the global fuel analysis as input. The uncertainty pertaining to the calculated temperature in state-of-theart modeling is around $10 \%$ at a confidence level of $95 \%$ $[10,32,33]$. In the present work, $10 \%( \pm 5 \%)$ of the calculated temperature is assumed as width of the temperature uncertainty band. The lower and upper bounds of this range are determined by multiplying - within the fission gas behavior model and at each time step - the calculated temperature by scaling factors of 0.95 and 1.05 , respectively. 
Temperature being a solution variable of the global fuel analysis, in turn affected by the calculated fission gas swelling and release (Section 1), a more rigorous study would consider the independent variables of temperature calculation (e.g., material properties) as sensitivity parameters. Also, applying the sensitivity range to the difference between the fuel centerline and the coolant temperatures, which is approximately proportional to the local linear heat rate, would be more physically meaningful. However, the above simple approach may be appropriate for an initial assessment of the role of temperature uncertainty in fission gas behavior modeling.

\subsection{Grain radius}

The average fuel grain radius increases during irradiation as grain growth takes place. This affects the fission gas behavior in three ways, i.e. [1,2,4,34]: (i) increasing the average diffusion distance for gas atoms generated in the grains, thus counteracting gas transport to the grain boundaries (Eq. 1), (ii) reducing the grain surface to volume ratio, hence the capacity of the grain faces to store fission gas and the grain-face swelling (Eq. 5), and (iii) through grain boundary sweeping which provides an additional mechanism for the collection of (largely insoluble) gas atoms at the grain boundaries.

In order to allow for the above effects, the present fission gas behavior model receives the grain radius calculated by a dedicated grain growth model as input. As a simple approach, temperature-dependent grain growth is accounted for using the model of Ainscough et al. [35]

$$
\frac{d l}{d t}=K\left(\frac{1}{l}-\frac{1}{l_{m}}\right)
$$

where $l(\mathrm{~m})$ is the 2-dimensional (linear intercept) average grain diameter, $K\left(\mathrm{~m}^{2} \mathrm{~s}^{-1}\right)$ the kinetic coefficient, which is $1.46 \cdot 10^{-8} \exp \left(-2.67 \cdot 10^{7} /(R T)\right)$ for $R=8.314$ $\mathrm{J} /(\mathrm{mol} \cdot \mathrm{K})$, and $l_{m}(\mathrm{~m})$ is the limiting linear intercept. The latter is a function of temperature such that $l_{m}=$ $2.23 \cdot 10^{-3} \exp (-7620 / T)$. The 3 -dimensional grain radius, $r_{g r}$, is obtained by multiplying $l$ by a factor of $1.56 / 2$ [36].

The fraction of intra-granular gas transferred to the grain boundaries during a time step through grain boundary sweeping, $f(-)$, is considered to equal the fraction of grain volume swept by the moving boundaries [4]

$f=\frac{r_{g r, i}^{3}-r_{g r, i-1}^{3}}{r_{g r, i}^{3}}$

where the indices $i-1$ and $i$ refer to the previous and current time step, respectively.

Experimental observations of the influence of grain radius on fission gas behavior include the work of Turnbull [37], which showed a significant reduction of both fission gas release and swelling with increasing grain size in $\mathrm{UO}_{2}$ specimens irradiated at $1750^{\circ} \mathrm{C}$ up to $0.4 \%$ FIMA. The experiments of Hastings et al. [38] indicated a significant contribution of grain boundary sweeping to gas transfer to grain boundaries in $\mathrm{UO}_{2}$ fuel power ramped to 55-65 $\mathrm{kW} \mathrm{m}^{-1}$. Tests of fuel rods with different grain size irradiated under similar conditions in the Halden reactor demonstrated that the grain radius had a a substantial effect on FGR [4].

Therefore, fission gas behavior calculations are affected by uncertainties in the calculated fuel grain radius. Van Uffelen et al. [39] reported different estimates for the grain growth kinetic coefficient in $\mathrm{UO}_{2}$, showing a scatter of about 3 orders of magnitude in the available data. Botazzoli [34] compared several $\mathrm{UO}_{2}$ grain growth models to a large amount of experimental data from the literature. His study pointed out that the ratio of predicted to experimental grain size is characterized by mean $\mu \approx 1$ and standard deviation $\sigma \approx 0.3$ for all considered models, including the model of Ainscough et al. [35] used in the present work (Eq. 7). Assuming a normal distribution for the calculated grain radius, the approximate uncertainty band is expressed here as the $95 \%$ confidence interval of $\pm 2 \sigma \approx \pm 0.6$. The lower and upper bounds of this range are determined by multiplying - within the fission gas behavior model and at each time step - the calculated grain radius by scaling factors of 0.4 and 1.6, respectively.

\subsection{Intra-granular gas atom diffusion coefficient}

Previous findings from the literature suggest that the uncertainty associated with the intra-granular (lattice) gas atom diffusion coefficient appearing in Eq. 1 plays an important role in limiting the accuracy of fission gas behavior predictions. White and Tucker [18] postulated that the main cause of the observed discrepancies between model and experiment in terms of fission gas and unstable fission product release lay in the uncertainties in the lattice diffusion coefficients. Simulations performed by Van Uffelen et al. [40] of power ramp and power cycling tests of $\mathrm{UO}_{2}$ fuel specimens from [27] showed variations up to a factor of 2 in the calculated FGR when considering the uncertainty in the intra-granular gas atom diffusion coefficient.

In the present fission gas behavior model, the following correlation is adopted as reference for calculating the intragranular gas atom diffusion coefficient $[15,41]$

$$
\begin{aligned}
& D_{i g}=D_{1}+D_{2} \\
& D_{1}=7.6 \times 10^{-10} \exp \left(-4.86 \times 10^{-19} /(k T)\right) \\
& D_{2}=1.41 \times 10^{-25} \sqrt{F} \exp \left(-1.91 \times 10^{-19} /(k T)\right)
\end{aligned}
$$

where $D_{1}\left(\mathrm{~m}^{2} \mathrm{~s}^{-1}\right)$ represents intrinsic thermal diffusion, $D_{2}\left(\mathrm{~m}^{2} \mathrm{~s}^{-1}\right)$ represents irradiation-enhanced diffusion, and $F\left(\mathrm{~m}^{-3} \mathrm{~s}^{-1}\right)$ is the fission rate.

The high uncertainty that pertains to the diffusion coefficient of fission gas atoms in the $\mathrm{UO}_{2}$ grains is well known. Lawrence [42] reviewed the experimental investigations of the lattice diffusion coefficient in $\mathrm{UO}_{2}$, revealing a very large dispersion in the results. The subsequent review of Matzke [43] demonstrated a scatter of about two 
orders of magnitude, depending on the considered temperature, between different experimental data sets including those from Matzke [44], Miekeley and Felix [45], and Carter et al. [46]. Prussin et al. [47] pointed out an uncertainty of at least two orders of magnitude for the lattice diffusion coefficient of $\mathrm{Xe}$ in $\mathrm{UO}_{2}$, based on various postirradiation annealing measurements including those from Matzke [44,48], Miekeley and Felix [45], Carter et al. [46], and Turnbull et al. [41]. Lattice diffusion coefficients of stable fission gases from White and Turnbull [49-51] measured in the Halden gas flow rigs also indicated a scatter of approximately two orders of magnitude, depending on the considered temperature, between different measurements. A spread of at least two orders of magnitude was pointed out by Olander and Van Uffelen [7] for the lattice diffusion coefficient of $\mathrm{Xe}$ in $\mathrm{UO}_{2}$ at $1400^{\circ} \mathrm{C}$, considering data from Davies and Long [52], Cornell [53], and Matzke [54]. Based on the above information, a range covering two orders of magnitude (factor of 100) is considered here to represent the uncertainty pertaining to the intra-granular gas atom diffusion coefficient. The lower and upper bounds of this range are determined by multiplying the reference value (Eq. 9) by scaling factors of 0.1 and 10, respectively. Coherently with the nature of the referenced experimental data, and in line with previous sensitivity analyses $[8,9,40]$, the uncertainty is applied to the diffusion coefficient rather than separately to prefactors and activation energies.

Note that the trapping parameter appearing in Eq. 1 is proportional to the intra-granular gas atom diffusion coefficient $[1,2,18,24,55]$. Such dependency is included in the present model, as detailed in [15,22]. Therefore, applying the considered uncertainty to the intra-granular diffusion coefficient also results in accounting for the corresponding uncertainty in the trapping parameter.

\subsection{Intra-granular resolution parameter}

The interpretation of the process of fission gas atom resolution from intra-granular bubbles is controversial [20, 56-60], with different models leading to widely different values for the resolution parameter appearing in Eq. 1. Nelson [56] developed a model of resolution by removal of single fission gas atoms at a time by elastic collisions with fission fragments or energetic primary knock-on atoms (homogeneous resolution). Turnbull [57] proposed that all gas in a bubble intersected by a fission fragment is returned to the lattice as single atoms (heterogeneous resolution). The review of Olander and Wongsawaeng [59] pointed out a difference of two orders of magnitude between the resolution parameters calculated using different models. In particular, they estimated a value of $10^{-5} \mathrm{~s}^{-1}$ for the homogeneous resolution parameter, and around $10^{-3} \mathrm{~s}^{-1}$ for the heterogeneous resolution parameter at a typical LWR fission rate $F=10^{19} \mathrm{~m}^{-3} \mathrm{~s}^{-1}$. Based on this information, a sensitivity range covering two orders of magnitude (factor of 100) is considered here. The lower and upper bounds of this range are obtained by multiplying the reference value by scaling factors of 0.1 and 10 , respectively.

The correlation for the reference value of the resolution parameter is chosen as follows

$b=10^{-23} F$

so that, with $F=10^{19} \mathrm{~m}^{-3} \mathrm{~s}^{-1}$, the lower and upper bounds of the sensitivity range correspond to the approximate values for homogeneous $\left(b=10^{-5} \mathrm{~s}^{-1}\right)$ and heterogeneous ( $b=10^{-3} \mathrm{~s}^{-1}$ ) resolution [59], respectively. The adoption of a purely fission-rate dependent correlation for the resolution parameter is in line with the work of Lösönen [9]. Such an approach also concurs with the results of molecular dynamics simulations of resolution from a Xe bubble in a $\mathrm{UO}_{2}$ grain [60], which indicated that the resolution rate is almost independent of the intra-granular bubble size.

\subsection{Grain-boundary diffusion coefficient}

The grain-face gas bubble development depends on the diffusion coefficient of vacancies along grain boundaries appearing in Eq. 3. The grain-boundary diffusion coefficients of gas atoms and vacancies may be reasonably assumed to be equal (e.g., [8,28]). White [19] obtained values for the grain-boundary diffusion coefficient based on experimental observations of grain-face bubbles in $\mathrm{UO}_{2}$ interpreted using the Speight and Beere [26] model. White's data fit (with $\delta_{g}=3.5 \cdot 10^{-10} \mathrm{~m}$ ) conforms to the following correlation

$$
D_{g b}=8.86 \times 10^{-6} \exp \left(-5.75 \times 10^{-19} /(k T)\right)
$$

where $D_{g b}$ is expressed in $\mathrm{m}^{2} \mathrm{~s}^{-1}$. Equation 11 is adopted as reference in the present model for calculating the grainboundary diffusion coefficient.

Table 1: Selected uncertain parameters of the fission gas behavior model with relative uncertainty ranges and corresponding scaling factors used in the sensitivity analysis.

\begin{tabular}{llllc}
\hline Parameter & Uncertainty range & \multicolumn{3}{c}{ Scaling factors } \\
& & Low & Ref. & High \\
\hline Temperature, $T$ & $\pm 5 \%$ & 0.95 & 1 & 1.05 \\
Grain radius, $r_{g r}$ & $\pm 60 \%$ & 0.4 & 1 & 1.6 \\
Intra-granular diffusion coefficient, $D_{i g}$ & factor of 100 & 0.1 & 1 & 10 \\
Intra-granular resolution parameter, $b$ & factor of 100 & 0.1 & 1 & 10 \\
Grain-boundary diffusion coefficient, $D_{g b}$ & factor of 100 & 0.1 & 1 & 10 \\
\hline
\end{tabular}


Olander and Van Uffelen [7] attributed to the Xe grainboundary diffusion coefficient an uncertainty similar to that in the intra-granular diffusion coefficient. The available data for the grain-boundary diffusion coefficient include the results of White [19] as well as those obtained by Reynolds and Burton [61] from analysis of $\mathrm{UO}_{2}$ creep and sintering measurements. The data span a range of approximately two orders of magnitude, depending on the considered temperature, which is consistent with the uncertainty associated with the intra-granular diffusion coefficient (Section 3.3). On this basis, the uncertainties in the intra-granular and grain-boundary diffusion coefficients are considered alike in the present work. Therefore, a sensitivity range covering two orders of magnitude (factor of 100) is assumed for the grain-boundary diffusion coefficient. The lower and upper bounds of this range are obtained by multiplying the reference value (Eq. 11) by scaling factors of 0.1 and 10 , respectively.

Table 1 summarizes the uncertain parameters and relative sensitivity ranges considered in the present work.

\section{Methodology}

\subsection{Software framework}

To perform the sensitivity analysis, the BISON fuel performance code was interfaced to the DAKOTA software on the INL's supercomputer Fission.

BISON [16] is a finite-element based, engineering-scale nuclear fuel performance code developed at INL. The code is applicable to both steady and transient fuel behavior and can be used to analyze 1D spherically symmetric, 2D axisymmetric or 3D geometries. BISON's governing relations consist of fully-coupled partial differential equations for thermo-mechanics and species conservation, and include constitutive laws for both nonlinear kinematics and nonlinear material behavior.

DAKOTA [17] is a software framework developed at Sandia National Laboratories that offers access to a broad range of iterative capabilities through a single, relatively simple interface with a simulator like BISON. DAKOTA can be used to perform parameter studies, uncertainty quantification, sensitivity analysis, calibration, and optimization.

\subsection{Setup of calculations}

Firstly, an extensive sensitivity analysis was performed based on a suitably simple model of a single fuel pellet. In addition, simulations were carried out of a LWR fuel rod irradiation experiment, in order to consolidate the conclusions of the single-pellet study based on a real fuel rod irradiation. The analyzed cases are briefly described in Subsections 4.2.1 and 4.2.2.

\subsubsection{Single-pellet model}

A $2 \mathrm{D}$ axisymmetric model of a single $\mathrm{UO}_{2}$ fuel pellet is considered. The assumed geometry is shown in Fig. 1. The dimensions follow the specifications of the simplified priority case 27(1) of the IAEA FUMEX-II Project [4], i.e., a fuel pellet with radius of $5.305 \mathrm{~mm}$ and height of $12.7 \mathrm{~mm}$ is considered. Cladding and coolant are not considered in this simple model. A $24 \times 16$ finite element mesh is adopted (Fig. 1), whose adequacy was demonstrated based on a mesh convergence study also involving calculations with $48 \times 32$ elements. $\mathrm{UO}_{2}$ fuel at $95 \%$ theoretical density (TD) with a fabricated grain radius of $5 \mu \mathrm{m}$ is considered. The linear heat rate (LHR) is assumed to rise linearly over three hours and is then held constant at a specified level. The BISON simulations allow for temperature and burn-up dependent fuel thermal conductivity, fuel thermal expansion, elasticity, densification and solid fission product swelling as detailed in [16], and fission gas release and swelling according to the model outlined in Section 2. The effect of hydrostatic stress on fission gas behavior is not considered.

In order to investigate the sensitivity of fission gas behavior calculations to the uncertain parameters for different situations covering a broad range of LWR operating conditions, different linear heat rates and time frames are considered. Specifically, the sensitivity analysis is performed for each of $5 \mathrm{LHR}$ values between 20 and $40 \mathrm{~kW} \mathrm{~m}^{-1}$ in $5 \mathrm{~kW} \mathrm{~m}^{-1}$ steps, and for irradiation times of $3,4.5$ and 6 years. It follows that the study considers 15 different conditions, covering fuel centerline temperatures from 1000 to $2000 \mathrm{~K}$, approximately, and a burn-up range from 27 to $110 \mathrm{GWd} \mathrm{t}_{\mathrm{U}}{ }^{-1}$, approximately.

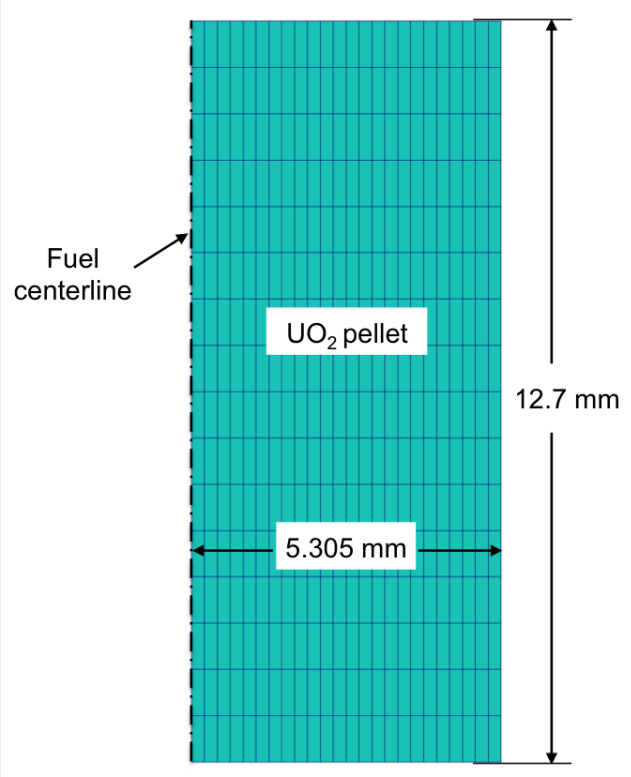

Figure 1: Geometry and mesh of the 2D axisymmetric singlepellet model. 


\subsubsection{Fuel rod irradiation experiment}

The GE7 irradiation experiment from the Ris $\varnothing-3$ experimental program, included in the IAEA FUMEX-III Project, is considered $[5,62,63]$. The as-fabricated fuel rod (ZX115) consisted of pellets of $\mathrm{UO}_{2}$ at $95.2 \%$ TD with 2dimensional average grain diameter of approximately 12 $\mu \mathrm{m}$, and Zircaloy-2 cladding with an outer diameter of $12.26 \mathrm{~mm}$. The experiment involved a fuel rod base irradiation up to about $40 \mathrm{GWd} \mathrm{t}_{\mathrm{U}}{ }^{-1}$ in the Quad Cities-1 BWR (USA), and subsequent power transient test to about 35 $\mathrm{kW} \mathrm{m}^{-1}$ in the DR3 research reactor at Ris $\varnothing$ (Denmark). The fabrication characteristics of the fuel rod are given in Table 2. More details on fuel fabrication data and irradiation conditions are reported in $[62,63]$. For the present simulations, the fuel rod was represented by a 2D axisymmetric model, with the pellet stack represented as a smeared fuel column. The calculations were carried out coherently with the power history and coolant conditions from the beginning of the base irradiation to the end of the transient test, using the pre-irradiation characterization data provided in $[62,63]$. The BISON simulations allow for the modeling aspects detailed in [16], including thermal expansion, elasticity, thermal and irradiation creep of both fuel and cladding, fuel densification, pellet-fragment relocation and solid fission product swelling, and cladding irradiation growth. Cladding plasticity, which may be of relevance during power transients, is taken into account by means of a von Mises plasticity model (e.g., [64]). Fission gas release and swelling are calculated using the model outlined in Section 2. The effect of hydrostatic stress on fission gas behavior is not considered.

Measurements of FGR and cladding outer diameter at the end of irradiation are available for the Ris $\varnothing-3$ GE7 experiment, which are compared to calculations in order to demonstrate a reasonably accurate representation of the relevant phenomena by the BISON code with the new fission gas behavior model.

\subsection{Sensitivity analysis techniques}

Relative to uncertainty quantification, which aims at obtaining probability distributions for the outputs given a set of distributions for the inputs, sensitivity analysis seeks to gain an understanding of the output range given a particular input domain, as well as to assess the importance of the different input parameters. The present work focuses on sensitivity analysis of fission gas behavior to the 5 sensitivity parameters specified in Section 3. Three levels are

Table 2: Fabrication data of the ZX115 fuel rod.

\begin{tabular}{ll}
\hline Pellet stack length $(\mathrm{mm})$ & 752.1 \\
Diametral gap $(\mathrm{mm})$ & 0.22 \\
Plenum length $(\mathrm{mm})$ & 143.4 \\
Internal free volume $\left(\mathrm{cm}^{3}\right)$ & 12 \\
Fill gas & $\mathrm{He}$ \\
Fill gas pressure $(\mathrm{MPa})$ & 0.29 \\
\hline
\end{tabular}

considered for each parameter, namely, the reference value and the lower and upper bounds of the uncertainty range (Table 1).

The simulations based on the $\mathrm{UO}_{2}$ single-pellet model cover all possible combinations of the LHR values and irradiation times specified in Section 4.2.1, and all possible combinations of parameter levels (low, reference, high). Hence, $3^{5}=243$ combinations ( 5 parameters at 3 levels each) are tested for each of the 15 considered LHR-time pairs ('conditions'). It follows that the sensitivity analysis involves a total of $15 \times 243=3645$ cases, each one corresponding to a specific BISON simulation. The output range for each condition is investigated, in order to gain an understanding of the results variability corresponding to the assumed parameter uncertainties (and their combination) for different operating situations. Moreover, in order to identify the input parameters that have the most significant influence on the output, a sensitivity analysis technique called main effects analysis and based on orthogonal array sampling (OAS) is used. This technique assesses the effect of an independent variable (input parameter) on a dependent variable (output quantity), averaging across the levels of the other independent variables (main effect) $[65,66]$.

The sensitivity analysis based on the Ris $\varnothing-3$ GE7 fuel rod experiment focuses on investigating the bounds of the variability range of the BISON predictions, over all possible combinations of levels for the 5 considered parameters of the fission gas behavior model.

The results of the study are discussed in Section 5 .

\section{Results and discussion}

The effects of the uncertain parameters on both fission gas release and swelling are investigated.

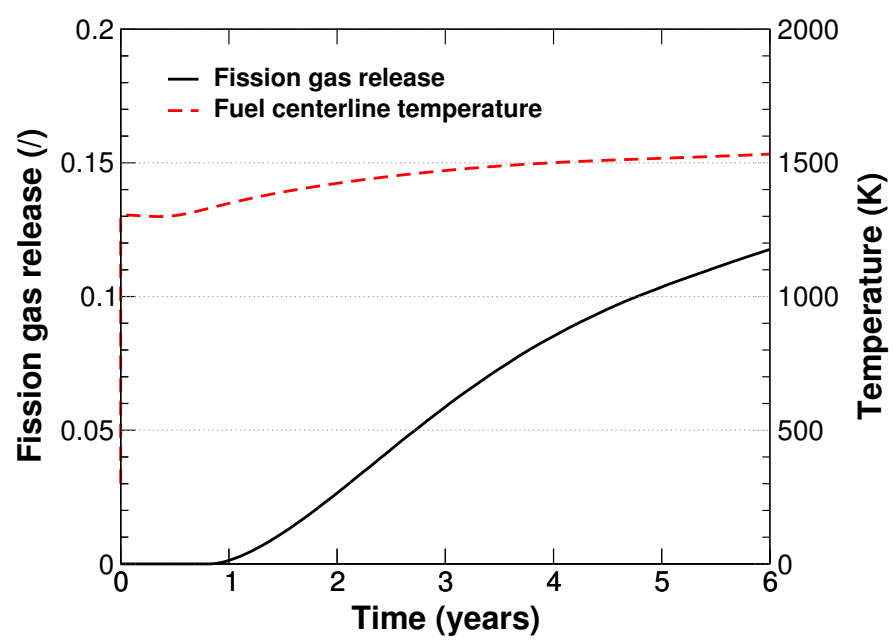

Figure 2: Fission gas release and fuel centerline temperature as a function of time for the reference single-pellet simulation with $\mathrm{LHR}=30 \mathrm{~kW} \mathrm{~m}^{-1}$. 

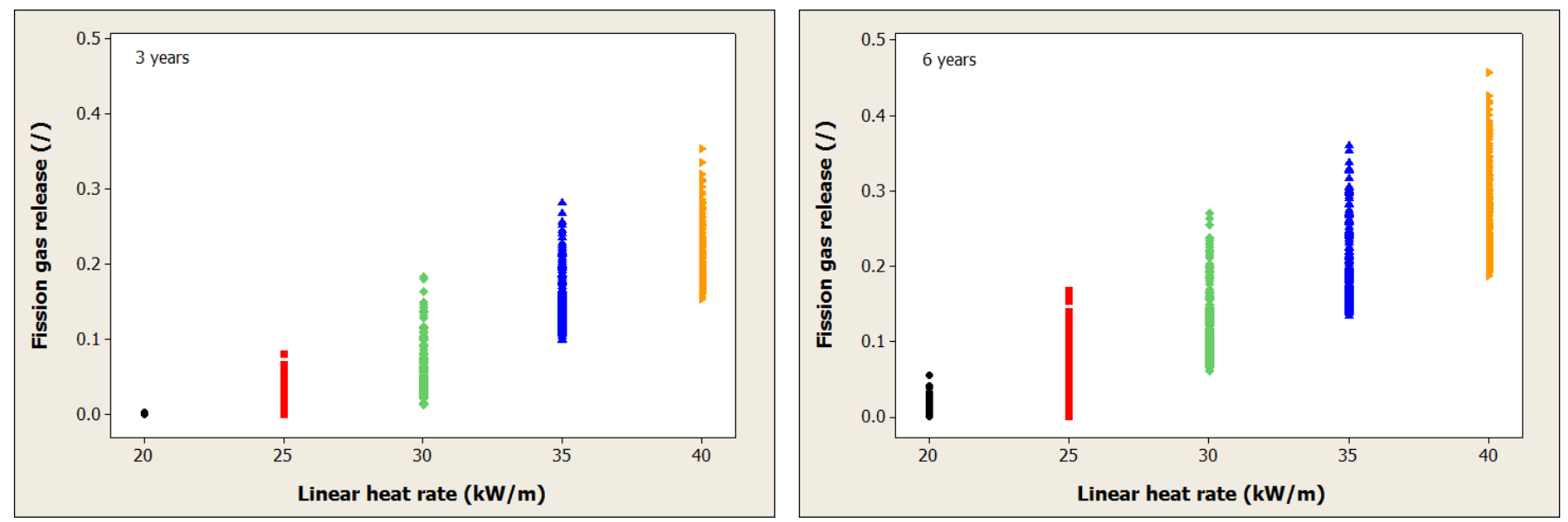

Figure 3: Plots of FGR scatter for the different LHR values and irradiation times of 3 years (left) and 6 years (right).

The quantities are computed for each integration point in the fuel finite element mesh, with the FGR defined as the ratio of the released to generated gas (fractional release), and the fission gas swelling defined as the fractional volume increment of the fuel. The integral FGR is considered in the following, which is obtained by summing the contributions from all integration points. The integral FGR is generally the quantity of interest to engineeringscale fuel modeling, as it corresponds to the total amount of gas released to the fuel rod free volume (Section 1).

\subsection{Fission gas release}

The time evolution of calculated FGR and fuel centerline temperature for the single-pellet simulation with all parameters set to the reference values is shown in Fig. 2 . A 6-year irradiation at $30 \mathrm{~kW} \mathrm{~m}^{-1}$ is considered. The temperature gradually increases during irradiation due to thermal conductivity degradation with increasing burn-up [16]. The incubation behavior of the FGR, which stems from the grain face saturation condition in the fission gas behavior model (Section 2.2), is evident.

Figure 3 illustrates the FGR results obtained, for each considered LHR value, with all different combinations of parameter levels specified in Section 4.3. The results for irradiation times of 3 and 6 years are shown. A remarkable scatter of the calculated FGR, due to the variation of the selected input parameters within the considered uncertainty ranges, is evident. For each of the considered time frames (3, 4.5 and 6 years), some combinations of parameter levels lead to zero values of calculated FGR at the lowest LHRs of 20 and $25 \mathrm{~kW} \mathrm{~m}^{-1}$, meaning that the grain face saturation condition for the onset of FGR is not attained during these calculations. Note that athermal FGR, which is independent of grain face saturation $[1,2,4,8]$, is not allowed for in this work. Considering all 3645 BISON simulations involved in the single-pellet sensitivity analysis, the calculated FGR covers a range from 0 to about $45 \%$.

\subsubsection{Variation factors analysis}

A more detailed assessment of the variability of the calculated FGR and its dependence on the simulated conditions is carried out through a study of FGR variation factors. A variation factor is defined here as the ratio of the highest to the lowest FGR obtained over all possible combinations of parameter levels, for a given LHR-time condition. The variation factor is intended as an indication of the output (FGR) range that corresponds to the considered input domain (combined uncertainty bands).

Variation factors for all LHR-time conditions considered in the present work (excluding those with a lower FGR bound of zero) are shown in Fig. 4, as a function of the mean of FGR results at the given condition. The plot points out a FGR variability of a factor of 2 and higher, resulting from the considered uncertainties in the parameters. More precisely, according to the present simple study, values of calculated FGR around $15 \%$ and higher are affected by an uncertainty of a factor of 2 to 3, approximately. Moreover, the variability of the results appears

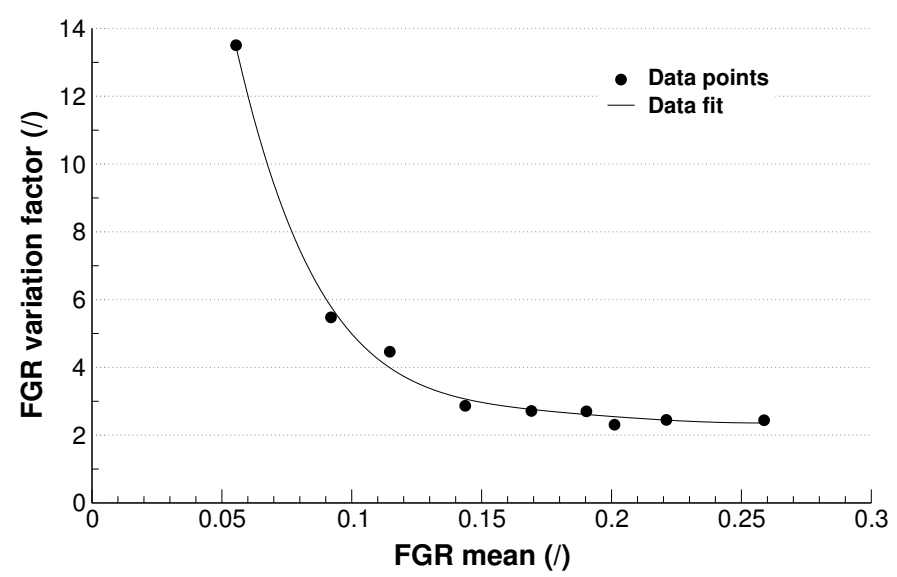

Figure 4: Variation factors of fission gas release over all possible combinations of parameter levels. Each data point corresponds to the simulations at a given LHR-time condition. 
to increase towards the lower FGR values. This circumstance may be ascribed to the increasing proximity to the threshold for the onset of thermal FGR (Section 2.2), so that small changes in the parameters lead to large factorial variations of FGR. The present results appear to be consistent with the known difficulty to predict low FGR values by means of fuel performance codes $[3,15]$.

Considering the simulation of an experiment, the variation factor analysis can provide an estimation of the maximum deviation of the calculated FGR from the experimental one. Assuming that the 'real' (experimental) value can lie anywhere within the variation range of the calculated values, the maximum deviation (maximum underand over-prediction) is equal to the variation factor. It follows that a variation factor of 2 in the calculated FGR corresponds to a maximum prediction error of a factor of 2 up and down. Therefore, the present sensitivity analysis partly supports the estimation of a factor of 2 up and down as a range of tolerance commonly associated with FGR predictions by means of fuel performance codes [3$5,10]$. In fact, such a deviation appears to represent a lower bound. A tolerance of a factor of 3 may also be considered as acceptable, and higher prediction errors may be expected for FGR values around $10 \%$ and lower. These considerations neglect the additional inaccuracy, which is independent of parameter uncertainties but associated with simplifying the complex physical processes into a model.

\subsubsection{Main effects analysis}

The relative importance of the single uncertain parameters in FGR calculations is investigated through main effects analysis (Section 4.3). Plots of main effects are presented in Fig. 5, which refers to an irradiation time of 3 years and two different LHR values of 30 and 40 $\mathrm{kW} \mathrm{m}^{-1}$. These plots are representative of the results obtained through the whole sensitivity analysis.

All considered uncertain parameters appear to have a significant effect. The highest influence is associated with the intra-granular diffusion coefficient and the resolution parameter. Also, the impact of the uncertainties in the calculated fuel temperature and in the grain-boundary diffusion coefficient is remarkable. Note that the diffusion coefficients and the grain radius are temperature-dependent, hence the effects of these parameters are partly due to the relative intrinsic uncertainties, and partly associated with temperature uncertainty (Section 3). A higher grain radius can result in either a lower or a higher FGR, depending on the case (Fig. 5). Such non-univocal correlation is associated with the multiple concurrent and opposing effects of grain size on FGR (Section 3.2).

The present results indicate that a better characterization of the intra-granular diffusion coefficient and resolution parameter may be considered as priority in terms of improving the accuracy of FGR predictions. To this end, advances may be achieved through both experimental research and theoretical approaches, such as atomistic modeling [67] and molecular dynamics techniques [60]. Also, the predictive capability of fuel performance codes in terms of FGR may be significantly enhanced if a reduction of the uncertainty pertaining to the calculated temperature is achieved.

The above results largely confirm the outcomes of the sensitivity analysis presented in [8] in terms of the uncertain parameters that mostly influence FGR calculations. Differently from [8], the present study does not allow for athermal release, which can provide a significant contribution to FGR at low temperature and will be considered in a future work.

\subsubsection{Irradiation experiment analysis}

BISON results for the simulations of the Ris $\varnothing-3$ GE7 irradiation experiment are presented in Fig. 6, showing fission gas release as a function of time during the transient test. Among all combinations of parameter levels specified in Section 4.3, only those that resulted in the lowest and highest calculated fission gas release at the end
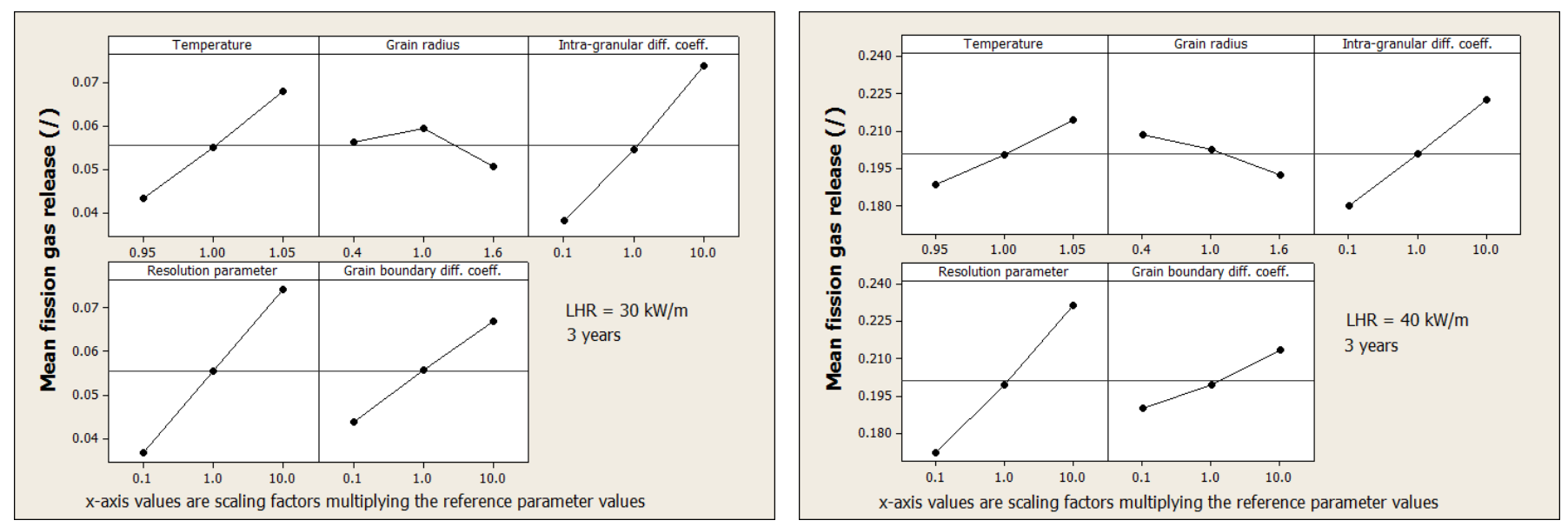

Figure 5: Main effects of parameters on calculated FGR for an irradiation time of 3 years and LHR values of $30 \mathrm{~kW} \mathrm{~m}^{-1}$ (left) and $40 \mathrm{~kW} \mathrm{~m}^{-1}$ (right). 


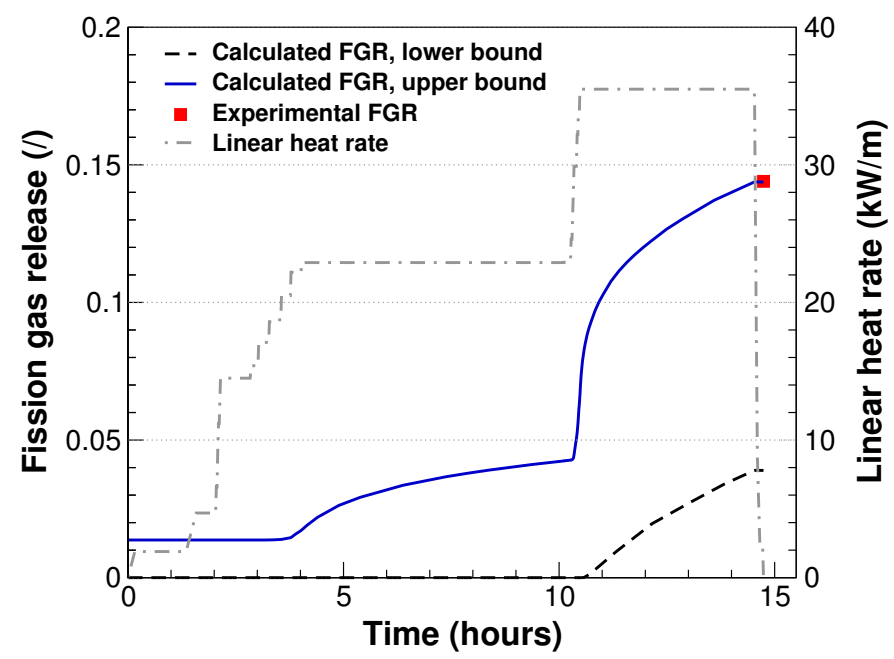

Figure 6: Fission gas release and rod average linear heat rate as a function of time for the Ris $\varnothing-3$ GE7 experiment. Both lower and upper bounds of the calculated FGR are shown along with the post-irradiation experimental value. Time zero corresponds to the beginning of the transient test.

of irradiation are included, in order to give an account of the output range. The post-irradiation experimental FGR value $[62,63]$ is also shown. The figure illustrates only the transient test following the base irradiation.

A variability of a factor of approximately 3.5 is observed in the calculated FGR at the end of irradiation, with the experimental value lying close to the upper bound of the variation range. Hence, the results of the irradiation experiment analysis appear to confirm the conclusions of the single-pellet study, in terms of the significant uncertainty in FGR predictions ensuing from the uncertainties in the model parameters. Also, the predictive accuracy of the BISON code with the new fission gas behavior model appears to be reasonable, considering the inherent modeling uncertainty.

\subsection{Fission gas swelling}

The radial profiles of calculated fission gas swelling, temperature and grain radius for the single-pellet simulation with all parameters set to the reference values are shown in Fig. 7. The plot refers to an irradiation time of 3 years at $30 \mathrm{~kW} \mathrm{~m}^{-1}$. The swelling decreases with increasing distance from the fuel centerline, reflecting the spatial dependence of fuel temperature. In fact, temperature drives both inflow of gas atoms and absorption of vacancies at the grain-face bubbles, thus affecting bubble development which determines fission gas swelling. Also, the coupling of the swelling with the FGR is taken into account by the present model, with gas release resulting in reduction of the amount of gas retained at the grain faces (Section 2). In fact, the irregularity observed in the swelling profile at a radial position of about $3 \mathrm{~mm}$ corresponds to the limit of the fuel zone affected by gas release
[15]. As shown in Fig. 7, the simulation involves significant temperature-driven grain growth in the central region of the fuel pellet, with $5 \mu \mathrm{m}$ being the initial grain radius.

\subsubsection{Main effects analysis}

In order to give an account of the relative importance of the uncertain parameters to the calculated local fission gas swelling, the main effects analysis is presented here for the swelling at fuel centerline. Plots of main effects are shown in Fig. 8, which refers to an irradiation time of 3 years and two different LHR values of 20 and $40 \mathrm{~kW} \mathrm{~m}^{-1}$. These plots are representative of the results obtained through the whole sensitivity analysis.

For the irradiation at moderate power of $20 \mathrm{~kW} \mathrm{~m}^{-1}$ (Fig. 8, left), the highest influence is associated with the intra-granular diffusion coefficient, which confirms the importance of the uncertainty relative to this parameter in fission gas behavior calculations. Moreover, the influence of the uncertainty in the calculated grain radius is remarkable. The grain radius determines the grain surface to volume ratio and directly affects the swelling through Eq. 5. For the irradiation at high power of $40 \mathrm{~kW} \mathrm{~m}^{-1}$ (Fig. 8, right), the uncertainty in the calculated grain radius appears to play a dominant role. Under such conditions, significant grain growth takes place in the central region of the fuel pellet, and FGR occurs. The latter counteracts gas atom and vacancy flow to the grain-face bubbles in determining the rate of fission gas swelling. It follows that the importance of the grain radius relative to the diffusion coefficients and resolution parameter increases. In the limiting situation of swelling saturation (Section 2.2), which may be reached after prolonged irradiation at high temperature, the swelling can vary (decrease) only due to grain growth (Eq. 5).

The present results suggest that the development of increasingly accurate grain growth models would be of im-

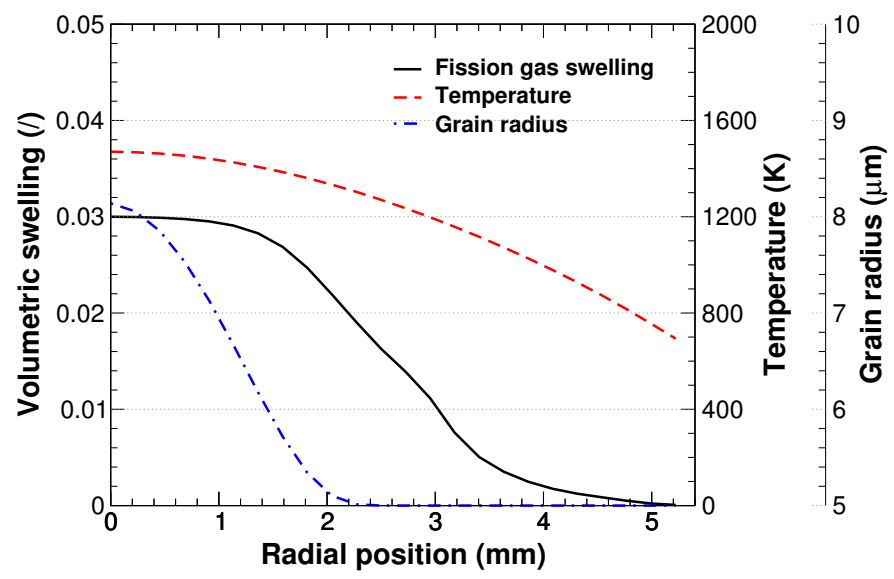

Figure 7: Fission gas swelling, temperature and grain radius as a function of the radial distance from the fuel centerline for the reference single-pellet simulation with $\mathrm{LHR}=30 \mathrm{~kW} \mathrm{~m}^{-1}$. The plot refers to an irradiation time of 3 years. 

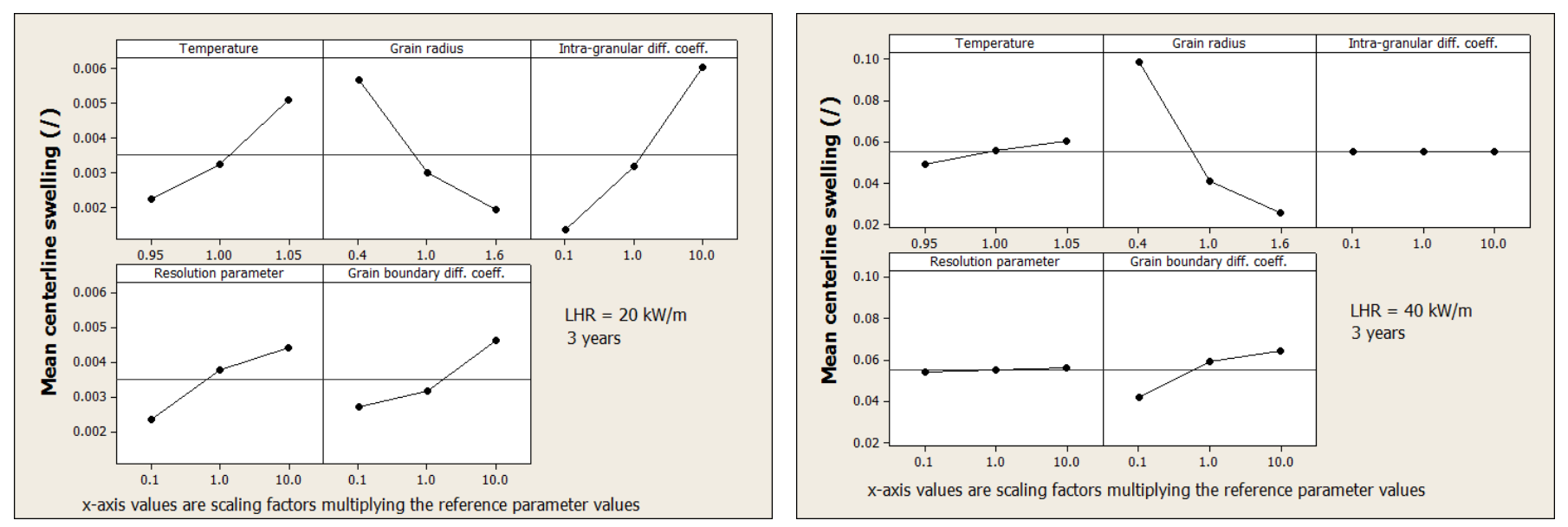

Figure 8: Main effects of parameters on calculated fission gas swelling at fuel centerline for an irradiation time of 3 years and LHR values of $20 \mathrm{~kW} \mathrm{~m}^{-1}$ (left) and $40 \mathrm{~kW} \mathrm{~m}^{-1}$ (right).

portance in terms of improving fission gas swelling predictions, especially during high temperature situations such as power transients. Lower-length scale modeling may lead to advances in this respect [68].

\subsubsection{Irradiation experiment analysis}

Under PCMI conditions, fission gas swelling along with thermal expansion of the fuel pellets can drive cladding stress and diametral strain $[1,5]$. Given the importance of fission gas swelling in the mechanical analysis of the cladding, investigating the related uncertainties is of interest from an engineering standpoint. Indeed, the calculated cladding strain depends on several processes, also includ-

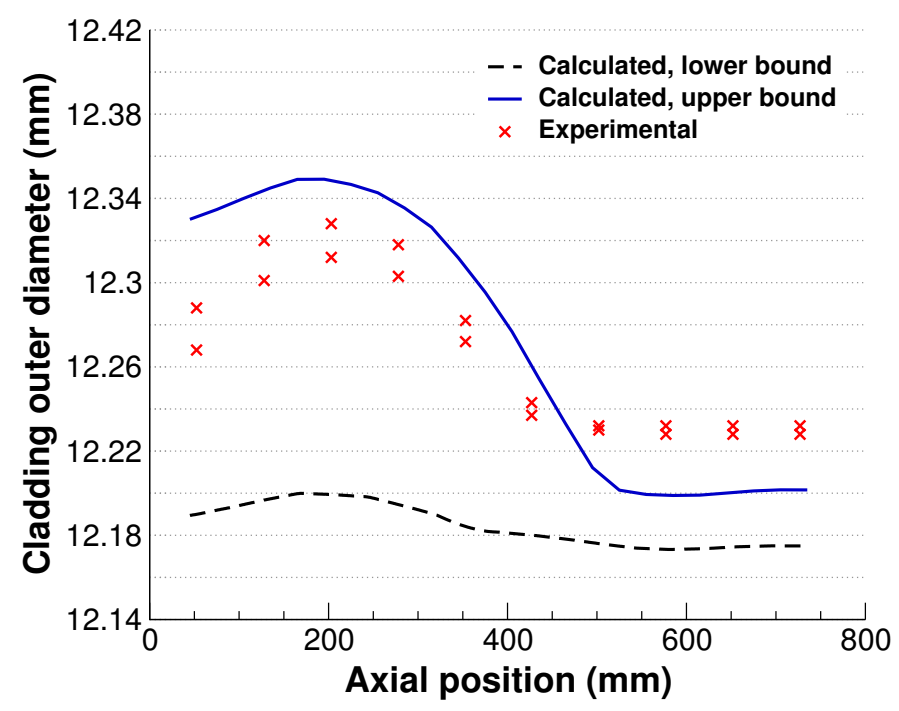

Figure 9: Cladding outer diameter as a function of the axial distance from the rod bottom for the Ris $\varnothing-3$ GE7 experiment at the end of irradiation. The results characterized by the lowest and highest cladding diameter are shown along with the postirradiation experimental data. The fabricated cladding outer diameter is $12.26 \mathrm{~mm}$. ing cladding thermal expansion, elasticity, creep and plasticity, and is therefore affected by uncertainties pertaining to different models. Here, a preliminary assessment is carried out of the effect of the sole fission gas behavior uncertainties on cladding diametral strain predictions. The Risø-3 GE7 experiment is considered, which comprises a power transient test involving PCMI.

The results of the BISON fuel rod simulations are presented in Fig. 9, showing the calculated cladding outer diameter at the end of irradiation as a function of axial position. Among all combinations of parameter levels specified in Section 4.3, only those that resulted in the lowest and highest cladding diameter are included, in order to give an account of the output range. The post-irradiation experimental data are also shown, which refer to measurements both at mid-pellet and pellet-end positions $[62,63]$. The significant role of the considered uncertainties in the analysis is evident. A maximum difference of about 150 $\mu \mathrm{m}$ is observed between the upper and lower bounds of the calculated cladding diameter. The experimental data lie in the vicinity of the upper bound of the variation range, thus indicating a reasonable accuracy of the BISON fuel rod thermo-mechanical analysis.

The present results confirm the importance of fission gas behavior modeling and the associated uncertainties to the assessment of cladding strain during power transients and PCMI. The results also indicate that the uncertainty in fission gas swelling calculations may partly explain the difficulty that fuel performance codes have predicting cladding diametral strain during power transients $[4,5]$.

\section{Conclusions}

In this paper, a sensitivity analysis was carried out in order to investigate the role of uncertainties in fission gas behavior calculations as part of engineering-scale nuclear fuel modeling. The BISON fuel performance code 
with a new physics-based model for fission gas release and swelling, and the DAKOTA uncertainty quantification and sensitivity analysis software were employed. Five parameters of the fission gas behavior model were varied within ranges representative of the relative uncertainties and consistent with the information in the open literature. The performed simulations included simplified single-pellet irradiations covering a broad range of LWR operating conditions, as well as a fuel rod irradiation experiment involving a power transient test.

The results indicated that a deviation between calculated and measured fission gas release by a factor of 2 approximately corresponds to the inherent modeling uncertainty at high fission gas release, whereas significantly higher deviations may be expected for values around $10 \%$ and lower. Moreover, a significant impact of fission gas behavior uncertainties on fuel rod analysis in terms of cladding diametral strain during a power transient was demonstrated. In order to improve fission gas behavior calculations in engineering-scale fuel modeling, a better characterization of the intra-granular gas atom diffusion coefficient may provide significant advances. Also, progress in the modeling of the intra-granular gas atom resolution and grain growth processes would be beneficial. Finally, significant improvements may be expected if a reduction of the uncertainty in the calculated fuel temperature is achieved.

The present study indicated that modeling of fission gas behavior for engineering purposes is subject to intrinsic uncertainties that may by far exceed some physical details. It is therefore questionable whether in general the predictive accuracy can be improved by increasing model complexity. A higher accuracy can be obtained for a limited number of cases by fitting the model parameters to the experimental data, which nevertheless would not improve the confidence in the predictions if the model is applied to different fuel designs or irradiation conditions. Incidentally, limiting the complexity of submodels implemented in fuel performance codes preserves internal code consistency, considering the simplifications and uncertainties involved in engineering-scale fuel modeling [11]. On the other hand, a better characterization of the parameters through experimental and theoretical research may reduce the uncertainty in fission gas behavior calculations and in the multiple related aspects of fuel performance analysis.

\section{Acknowledgments}

GP wishes to thank Prof. Klaus Lassmann for some of the concepts that inspired this work.

This work was funded by the DOE Nuclear Energy Advanced Modeling and Simulation Program. The submitted manuscript has been authored by a contractor of the US Government under Contract DE-AC07-05ID14517. Accordingly, the US Government retains a non-exclusive, royalty free license to publish or reproduce the published form of this contribution, or allow others to do so, for US Government purposes.

\section{References}

[1] D.R. Olander, Fundamental Aspects of Nuclear Reactor Fuel Elements, Technical Information Center - Energy Research and Development Administration, University of California, Berkeley, CA, USA, 1976.

[2] P. Van Uffelen, R.J.M. Konings, C. Vitanza, J. Tulenko, Analysis of reactor fuel rod behavior, in: D.G. Cacuci (Ed.), Handbook of Nuclear Engineering, Vol. 13, Springer Science + Business Media, LLC., New York, NY, USA, 2010, pp. 1519-1627.

[3] Fuel modelling at extended burnup, Tech. Rep. IAEATECDOC-998 (1998).

[4] Fuel modelling at extended burnup (FUMEX-II), Tech. Rep. IAEA-TECDOC-1687 (2012).

[5] Improvement of computer codes used for fuel behaviour simulations (FUMEX-III), Tech. Rep. IAEA-TECDOC-1697 (2013).

[6] K. Lassmann, H. Benk, Numerical algorithms for intragranular fission gas release, Journal of Nuclear Materials 280 (2000) 127135 .

[7] D.R. Olander, P. Van Uffelen, On the role of grain boundary diffusion in fission gas release, Journal of Nuclear Materials 288 (2001) 137-147.

[8] P. Van Uffelen, Contribution to the modelling of fission gas release in light water reactor fuel, Ph.D. thesis, University of Liège, Belgium (2002).

[9] P. Lösönen, Modelling intragranular fission gas release in irradiation of sintered $\mathrm{LWR} \mathrm{UO}_{2}$ fuel, Journal of Nuclear Materials 304 (2002) 29-49.

[10] L.C. Bernard, J.L. Jacoud, P. Vesco, An efficient model for the analysis of fission gas release, Journal of Nuclear Materials 302 (2002) 125-134.

[11] K. Lassmann, The structure of fuel element codes, Nuclear Engineering and Design 57 (1980) 17-39.

[12] C. Calvin, D. Nowak, High performance computing in nuclear engineering, in: D.G. Cacuci (Ed.), Handbook of Nuclear Engineering, Vol. 12, Springer Science + Business Media, LLC., New York, NY, USA, 2010, pp. 1449-1517.

[13] A. Karahan, M.S. Kazimi, On the significance of modeling nuclear fuel behavior with the right representation of the physical phenomena, Nuclear Engineering and Design 241 (2011) 484491.

[14] K. Lassmann, TRANSURANUS: a fuel rod analysis code ready for use, Journal of Nuclear Materials 188 (1992) 295-302.

[15] G. Pastore, L. Luzzi, V. Di Marcello, P. Van Uffelen, Physicsbased modelling of fission gas swelling and release in $\mathrm{UO}_{2}$ applied to integral fuel rod analysis, Nuclear Engineering and Design 256 (2013) 75-86.

[16] R.L. Williamson, J.D. Hales, S.R. Novascone, M.R. Tonks, D.R. Gaston, C.J. Permann, D. Andrs, R.C. Martineau, Multidimensional multiphysics simulation of nuclear fuel behavior, Journal of Nuclear Materials 423 (2012) 149-163.

[17] B.M. Adams, K.R. Dalbey, M.S. Eldred, L.P. Swiler, W.J. Bohnhoff, J.P. Eddy, D.M. Vigil, P.D. Hough, S. Lefantzi, DAKOTA: A multilevel parallel object-oriented framework for design optimization, parameter estimation, uncertainty quantification, and sensitivity analysis. Version 5.2 user's manual, Tech. Rep. SAND2010-2183 (2011).

[18] R.J. White, M.O. Tucker, A new fission-gas release model, Journal of Nuclear Materials 118 (1983) 1-38.

[19] R.J. White, The development of grain-face porosity in irradiated oxide fuel, Journal of Nuclear Materials 325 (2004) 61-77.

[20] P. Garcia, G. Martin, C. Sabathier, G. Carlot, A. Michel, P. Martin, B. Dorado, M. Freyss, M. Bertolus, R. Skorek, J. Noirot, L. Noirot, O. Kaitasov, S. Maillard, Nucleation and growth of intragranular defect and insoluble atom clusters in nuclear oxide fuels, Nuclear Instruments and Methods in Physics Research B 277 (2012) 98-108.

[21] D.M. Perez, R.L. Williamson, S.R. Novascone, T.K. Larson, J.D. Hales, B.W. Spencer, G. Pastore, An evaluation of the nuclear fuel performance code BISON, in: Proc. of the Int. Conference on Mathematics and Computational Methods Applied 
to Nuclear Science and Engineering, Sun Valley, ID, USA, May 5-9, 2013

[22] G. Pastore, J.D. Hales, S.R. Novascone, D.M. Perez, B.W Spencer, R.L. Williamson, Analysis of fission gas release in LWR fuel using the BISON code, in: Proc. of the LWR Fuel Performance Meeting, Charlotte, NC, USA, September 15-19, 2013.

[23] J.D. Hales, R.L. Williamson, S.R. Novascone, D.M. Perez, B.W. Spencer, G. Pastore, Multidimensional multiphysics simulation of TRISO particle fuel, Journal of Nuclear Materials 443 (2013) $531-543$.

[24] M.V. Speight, A calculation on the migration of fission gas in material exhibiting precipitation and re-solution of gas atoms under irradiation, Nuclear Science and Engineering 37 (1969) $180-185$.

[25] M.S. Veshchunov, Modelling of grain face bubbles coalescence in irradiated $\mathrm{UO}_{2}$ fuel, Journal of Nuclear Materials 374 (2008) 44-53.

[26] M.V. Speight, W. Beere, Vacancy potential and void growth on grain boundaries, Metal Science 9 (1975) 190-191.

[27] R.J. White, R.C. Corcoran, P.J. Barnes, A summary of swelling data obtained from the AGR/Halden Ramp Test Programme, Tech. Rep. R\&T/NG/EXT/REP/0206/02 (2006).

[28] T. Kogai, Modelling of fission gas release and gaseous swelling of light water reactor fuels, Journal of Nuclear Materials 244 (1997) 131-140

[29] A.R. Massih, K. Forsberg, Calculation of grain boundary gaseous swelling in $\mathrm{UO}_{2}$, Journal of Nuclear Materials 377 (2008) 406-408.

[30] C. Vitanza, U. Graziani, N.T. Fordestrommen, K. Vilpponen, Fission gas release from in-pile measurements, Tech. Rep. HPR221.10 (1978).

[31] S. Kashibe, K. Une, Effect of external restraint on bubble swelling in $\mathrm{UO}_{2}$ fuels, Journal of Nuclear Materials 247 (1997) $138-146$.

32] P. Chantoin, J.A. Turnbull, W. Wiesenack, How good is fue modelling at extended burn-up?, Nuclear Engineering International 42 (518) (1997) 32-360.

[33] A. Bouloré, C. Struzik, F. Gaudier, Uncertainty and sensitivity analysis of the nuclear fuel thermal behavior, Nuclear Engineering and Design 253 (2012) 200-210.

[34] P. Botazzoli, Helium production and behaviour in LWR nuclear oxide fuels, Ph.D. thesis, Politecnico di Milano, Italy (2011).

[35] J.B. Ainscough, B.W. Oldfield, J.O. Ware, Isothermal grain growth kinetics in sintered $\mathrm{UO}_{2}$ pellets, Journal of Nuclear Materials 49 (1973) 117-128.

[36] M.I. Mendelson, Average grain size in polycrystalline ceramics, Journal of the American Ceramic Society 52 (1969) 443-446.

[37] J.A. Turnbull, The effect of grain size on the swelling and gas release properties of $\mathrm{UO}_{2}$ during irradiation, Journal of Nuclear Materials 50 (1974) 62-68.

[38] I.J. Hastings, A.D. Smith, P.J. Fehrenbach, T.J. Carter, Fission gas release from power-ramped $\mathrm{UO}_{2}$ fuel, Journal of Nuclear Materials 139 (1986) 531-543.

[39] P. Van Uffelen, P. Botazzoli, L. Luzzi, S. Bremier, A. Schubert, P. Raison, R. Eloirdi, M.A. Barker, An experimental study of grain growth in mixed oxide samples with various microstructures and plutonium concentrations, Journal of Nuclear Materials 434 (2013) 287-290.

[40] P. Van Uffelen, G. Pastore, V. Di Marcello, L. Luzzi, Multiscale modelling for the fission gas behavior in the TRANSURANUS code, Nuclear Engineering and Technology 43 (6) (2011) 477 488.

[41] J.A. Turnbull, C.A. Friskney, J.R. Findlay, F.A. Johnson, A.J Walter, The diffusion coefficients of gaseous and volatile species during the irradiation of uranium dioxide, Journal of Nuclear Materials 107 (1982) 168-184.

[42] G.T. Lawrence, A review of the diffusion coefficient of fissionproduct rare gases in uranium dioxide, Journal of Nuclear Materials 71 (1978) 195-218.

[43] H. Matzke, Gas release mechanisms in $\mathrm{UO}_{2}$ - a critical review, Radiation Effects 53 (1980) 219-242.
44 H. Matzke, Mobility and precipitation of rare gases in solids during annealing following ion bombardment, in: B. Navinsek (Ed.), Physics of ionized gases, Proc. Int. Summer School Hercegnovi, Inst. Josef Stefan, Ljubljana, Yugoslavia, 1970, p. 354.

[45] W. Miekeley, F. Felix, Effect of stoichiometry on diffusion of xenon in $\mathrm{UO}_{2}$, Journal of Nuclear Materials 42 (1972) 297-306.

[46] J.C. Carter, E.J. Driscoll, T.S. Elleman, Xenon-133 diffusion and trapping in single-crystal uranium dioxide, Physica Status Solidi A 14 (1972) 673-680.

[47] S.G. Prussin, D.R. Olander, W.K. Lau, L. Hansson, Release of fission products (Xe, I, Te, Cs, Mo and Tc) from polycrystalline $\mathrm{UO}_{2}$, Journal of Nuclear Materials 154 (1988) 25-37.

[48] H. Matzke, Diffusion in doped $\mathrm{UO}_{2}$, Nuclear Applications 2 (1966) 131-137.

[49] R.J. White, J.A. Turnbull, Fission product release measurements in the gas flow rigs and comparisons with data from UK sources, in: Enlarged Halden Programme Group Meeting, Sanderstölen, Norway, March 3-6, 1986.

[50] R.J. White, A new mechanistic model for the calculation of fission gas release, in: Proc. of the Int. Topical Meeting on Light Water Reactor Fuel Performance, West Palm Beach, FL, USA, April 17-21, 1994, pp. 196-202.

51] R.J. White, The fractal nature of the surface of uranium dioxide: a resolution of the short-lived/stable gas release dichotomy, Journal of Nuclear Materials 295 (2001) 133-148.

[52] D. Davies, G. Long, AERE Report 4347, Atomic Energy Research Establishment, Harwell (1963).

[53] R.M. Cornell, The growth of fission gas bubbles in irradiated uranium dioxide, Philosophical Magazine 19 (1969) 539-554.

[54] H. Matzke, Atomic mechanisms of mass transport in ceramic nuclear fuel materials, Journal of the Chemical Society, Faraday Transactions 86 (1990) 1243-1256.

[55] F.S. Ham, Theory of diffusion-limited precipitation, Journal of Physics and Chemistry of Solids 6 (1958) 335-351.

[56] R.S. Nelson, The stability of gas bubbles in an irradiation environment, Journal of Nuclear Materials 31 (1969) 153-161.

[57] J.A. Turnbull, The distribution of intragranular fission gas bubbles in $\mathrm{UO}_{2}$ during irradiation, Journal of Nuclear Materials 38 (1971) 203-212.

[58] P. Lösönen, On the behaviour of intragranular fission gas in $\mathrm{UO}_{2}$ fuel, Journal of Nuclear Materials 280 (2000) 56-72.

[59] D.R. Olander, D. Wongsawaeng, Re-solution of fission gas A review: Part I. Intragranular bubbles, Journal of Nuclear Materials 354 (2006) 94-109.

[60] K. Govers, C.L. Bishop, D.C. Parfitt, S.E. Lemehov, M. Verwerft, R.W. Grimes, Molecular dynamics study of Xe bubble re-solution in $\mathrm{UO}_{2}$, Journal of Nuclear Materials 420 (2012) $282-290$.

[61] G.L. Reynolds, B. Burton, Grain-boundary diffusion in uranium dioxide: the correlation between sintering and creep and a reinterpretation of creep mechanism, Journal of Nuclear Materials 82 (1979) 22-25.

[62] The Third Ris $\varnothing$ Fission Gas Project: Bump Test GE7 (ZX115), Tech. Rep. RISØ-FGP3-GE7 (1990).

63] E. Sartori, J. Killeen, J.A. Turnbull, International Fuel Performance Experiments (IFPE) database, OECD-NEA, 2010, available at http://www.oecd-nea.org/science/fuel/ifpelst.html.

[64] F. Dunne, N. Petrinic, Introduction to Computational Plasticity, Oxford University Press, Oxford, 2005.

[65] A.S. Hedayat, N.J.A. Sloane, J. Stufken, Orthogonal Arrays: Theory and Applications, Springer Series in Statistics, SpringerVerlag, New York, NY, 1999.

[66] D.C. Montgomery, G.C. Runger, Applied Statistics and Probability for Engineers, 5th edition, John Wiley \& Sons, Inc., 2011.

[67] D.A. Andersson, P. Garcia, X.-Y. Liu, G. Pastore, M. Tonks, P. Millett, B. Dorado, D.R. Gaston, D. Andrs, R.L. Williamson, R.C. Martineau, B.P. Uberuaga, C.R. Stanek, Atomistic modeling of intrinsic and radiation-enhanced fission gas (Xe) diffusion in $\mathrm{UO}_{2 \pm x}$ : Implications for nuclear fuel performance modeling, Journal of Nuclear Materials 451 (2014) 225-242. 
[68] M.R. Tonks, Y. Zhang, S.B. Biner, P.C. Millett, X. Bai, Guidance to design grain boundary mobility experiments with molecular dynamics and phase-field modeling, Acta Materialia 61 (2013) 1373-1382. 